\title{
Low-Complexity Transmit Antenna Selection in Large-Scale Spatial Modulation Systems
}

\author{
Peng Wei, ${ }^{1}$ Lu Yin, ${ }^{1}$ Yue Xiao, ${ }^{1,2} \mathrm{Xu} \mathrm{He},{ }^{1}$ and Shaoqian $\mathrm{Li}^{1}$ \\ ${ }^{1}$ National Key Laboratory of Science and Technology on Communications, University of Electronic Science and Technology of China, \\ Chengdu 611731, China \\ ${ }^{2}$ National Mobile Communications Research Laboratory, Southeast University, Nanjing 210018, China
}

Correspondence should be addressed to Yue Xiao; xiaoyue@uestc.edu.cn

Received 26 August 2015; Accepted 10 November 2015

Academic Editor: Periklis Chatzimisios

Copyright (C) 2015 Peng Wei et al. This is an open access article distributed under the Creative Commons Attribution License, which permits unrestricted use, distribution, and reproduction in any medium, provided the original work is properly cited.

\begin{abstract}
Transmit antenna selection (TAS) is an efficient way for improving the system performance of spatial modulation (SM) systems. However, in the case of large-scale multiple-input multiple-output (MIMO) configuration, the computational complexity of TAS in large-scale SM will be extremely high, which prohibits the application of TAS-SM in a real large-scale MIMO system for future 5G wireless communications. For solving this problem, in this paper, two novel low-complexity TAS schemes, named as norm-angle guided subset division (NAG-SD) and threshold-based NAG-SD ones, are proposed to offer a better tradeoff between computational complexity and system performance. Simulation results show that the proposed schemes can achieve better performance than traditional TAS schemes, while effectively reducing the computational complexity in large-scale spatial modulation systems.
\end{abstract}

\section{Introduction}

Recently, spatial modulation (SM) [1-3] was proposed as an efficient and low-complexity implementation of multipleinput multiple-output (MIMO) wireless systems. In SMMIMO, only one transmit antenna is activated at each single time slot, which introduces some special advantages in system design. Most of all, SM-MIMO can be applied in a single radio frequency (RF) system by switching the transmit antennas, which efficiently reduces the implement cost of MIMO systems, especially for massive MIMO $[4,5]$ with large number of transmit antennas. In general, largescale SM-MIMO has the potentials to be utilized in future 5G wireless communications, such as downlink massive MIMO [6], relay [7] and full duplex [8] assisted base station, and millimeter-wave communication systems [9], to improve the Quality-of-experience (QoE) of future wireless systems [10].

In the receiver of SM systems, maximum-likelihood (ML) detection algorithm [11] was proposed to obtain optimal system performance. For further improving the system performance under limited feedback, link adaptation for changing the transceiver parameters was developed such as in [11-17]. Among current link adaptation schemes, transmit antenna selection (TAS) $[12,13]$ is a class of efficient schemes, to fully explore the extra antenna resource for improving the performance.

For SM-MIMO link adaptation including TAS, the optimal criterion is to design the link by maximizing the minimum Euclidean distance (ED) among the legitimate transmit vectors $[12,13]$. However, the ED-based criterion will consume large complexity for an exhaustive search. In $[14,15]$, some simplified ED-based algorithms were proposed with lower complexity. In general, in the case of large-scale transmit antennas, ED-based criterion is extremely difficult and unpractical.

To further reduce the complexity of ED-based algorithms, some candidate TAS criterions were developed such as in [12, $16,17]$. More specifically, in [12], capacity optimized antenna selection (COAS) was proposed, where the antennas are selected with the maximum Frobenius norm of the columns in the channel matrix. COAS is with low complexity but its performance is not comparable to ED-based criterions. On the other hand, motivated by reference [18] which deals with the angles between transmit vectors, the correlation of 
the angles is considered as another TAS criterion [16, 17]. For example, antenna selection based on antenna correlation (AS-AC) was developed in [17]. However, the computational complexity for searching the best antenna correlations in [17] is high, and the performance is still not comparable to the optimal criterion.

For alleviating the high complexity of traditional TAS methods, in this paper, we propose a novel low-complexity TAS scheme norm-angle guided subset division (NAG-SD). The new TAS criterion is proposed considering not only the Frobenius norm of the columns in the channel matrix, but also the angles between the column vectors. We will show that the proposed scheme can achieve preferable performance compared to conventional TAS schemes, with considerable complexity for large-scale SM. Furthermore, based on the proposed NAG-SD scheme, a preset threshold is introduced for further reducing the computational complexity. We will show the so-called threshold-based NAG-SD scheme can offer a balanced tradeoff between system performance and computational complexity.

The rest of the paper is organized as follows. Section 2 outlines the system and signal model and summarizes the several conventional TAS schemes in spatial modulation. The proposed TAS algorithms are introduced in Section 3, and Section 4 reports simulation results. Finally, Section 5 concludes the paper.

\section{Conventional SM and TAS Schemes}

Assume a SM-MIMO system with $N_{t}$ transmit and $N_{r}$ receive antenna over flat Rayleigh fading channel. If $N_{s}$ transmit antennas are selected out of $N_{t}$ ones, the antenna subset, containing $N_{s} \leq N_{t}$ transmit antennas, is determined by a TAS criterion carried out at the receiver side. The receiver decides the selected subset $C_{i}^{N_{s} \times 1} \in S, i=0,1, \ldots, I$, where $S$ is the set of all possible $I=C_{N_{t}}^{N_{s}}$ subsets of the transmit antennas. Then the receiver will inform the transmitter about the indexes of the selected antenna subset.

At each time slot, data bits are mapped into the transmit vector as

$$
\mathbf{x}=s_{m} \mathbf{e}_{l} \in C^{N_{s} \times 1},
$$

where the complex-valued $s_{m}, m=0,1, \ldots, M-1$, is chosen from $M$-ary quadrature amplitude modulation ( $M$-QAM) constellations, and $\mathbf{e}_{l} \in C^{N_{s} \times 1}, l=0,1, \ldots, L-1$, is with only one none-zero value 1 , selected from the $N_{s}$-dimension vectors. In general, the transmit vector $\mathbf{x}$, which represents $\log _{2} N_{s} M$ bits, is transmitted over $N_{s}$ transmit antennas. At the receiver, the received signal vector can be expressed as

$$
\mathbf{y}=\mathbf{H}(i) \mathbf{x}+\mathbf{n},
$$

where $\mathbf{H}(i)$ is the channel matrix with $i=0,1, \ldots, I$, the channel fading coefficient between the $l$ th transmit and the $j$ th receive antenna, denoted by $h_{j l}$, is assumed to be independent identically distributed (i.i.d.) complex Gaussian random variables with zero mean and unit variance, that is, $C N(0,1)$, and the elements of $N_{r}$-dimensional noise vector $\mathbf{n}$ are Gaussian random variables obeying $C N\left(0, \sigma^{2}\right)$, where $\sigma^{2}$ is the power of the noise.

Using the ML detection criterion, the optimal estimate of the transmit symbol vector can be given by

$$
\widehat{\mathbf{x}}=\arg \min _{\mathbf{x} \in \Lambda}\|\mathbf{y}-\mathbf{H}(i) \mathbf{x}\|_{F}^{2},
$$

where $\Lambda$ is the set of all possible transmitted symbols.

However there are many TAS algorithms for SM-MIMO, such as ED-based [12, 13] as mentioned in Section 1, which consumes large complexity for an exhaustive search. Moreover, several simplified ED-based TAS algorithms have been developed for reducing the complexity, such as Euclid distance optimized antenna selection (EDAS) [14], EDAS reduced-low-complexity (RLC) [2], and singular value decomposition (SVD) EDAS [15]. Since the number of transmit antennas in large MIMO is massive, these algorithms are unpractical in high complexity. Furthermore, COAS [14] is with low complexity but its performance is not comparable to ED-based criterions and hence cannot be directly applied for large-scale SM. Thus, it is necessary to develop new schemes which make a balanced tradeoff between complexity and performance. In the next subsection, we expound two typical conventional TAS schemes, as ED-based and COAS, emphasized on performance and complexity, respectively.

2.1. ED-Based TAS. For SM-MIMO with ML detection, the performance of the receiver is dominated by the minimum Euclidean distance, which is defined as

$$
d_{\min }(\mathbf{H}(i))=\min _{\mathbf{x}_{j}, \mathbf{x}_{k} \in \Lambda, \mathbf{x}_{j} \neq \mathbf{x}_{k}}\left\|\mathbf{H}(i)\left(\mathbf{x}_{j}-\mathbf{x}_{k}\right)\right\|_{F},
$$

where $\mathbf{x}_{j}$ is one of the constellation of transmitted symbols sets $\Lambda$. Then, the suboptimal TAS scheme is to select the antenna subset which maximizes the minimum Euclidean distance and can be formulated as

$$
\widehat{\mathbf{H}}=\arg \max _{p \in P} d_{\min }\left(\mathbf{H}_{p}(i)\right),
$$

where $P$ is the candidate subsets of all possible transmit antenna combinations and $\mathbf{H}_{p}(i)$ is one of the alternative channel matrix subsets. This criterion gives the optimal performance in terms of bit error rate (BER). However, it desires an exhaustive search with high computational complexity especially for large QAM modulation constellations. Several simplified ED-based TAS algorithms have been developed for reducing the complexity, such as EDAS [12], EDAS-RLC [13], and SVD EDAS [15].

2.2. Capacity Optimized Antenna Selection. COAS algorithm was proposed in [12] as an efficient TAS criterion, where the antennas corresponding to the columns of the channel matrix with the maximum Frobenius norm are selected. The algorithm can be expressed as

$$
p=\arg \max _{\forall i, l \in\left\{1,2, \ldots, N_{t}\right\}}\left\|\mathbf{h}_{l}(i)\right\|_{F}^{2},
$$


where $\mathbf{h}(\cdot)$ is the column of channel matrix $\mathbf{H}(\cdot)$. To be specific, $\mathbf{h}_{l}(i)$ is the $l$ th column of the matrix $\mathbf{H}(i)$. $p$ represents the desired subset of antennas. COAS criterion is with much lower complexity compared to ED based one, at the cost of considerable performance loss.

\section{Proposed TAS Schemes for Large-Scale SM-MIMO}

3.1. NAG-SD Scheme. According to current literature, it is a challenge issue to design a TAS criterion offering a balanced tradeoff between performance and complexity. In this paper, based on traditional COAS and angle-based criterions, a hybrid TAS criterion NAG-SD is proposed which considers both the norms and the angles of the transmit SM-MIMO signal vectors. The proposed criterion aims at selecting the optimized channel matrix which increases the angles of the transmit vectors as well as the channel gains. Before expounding our proposed algorithm, we give the definition of vector angle as

$$
\begin{aligned}
& \cos \theta_{j, k}=\frac{\mathbf{h}_{j}^{\mathrm{H}} \mathbf{h}_{k}}{\left\|\mathbf{h}_{j}\right\|_{F}\left\|\mathbf{h}_{k}\right\|_{F}}, \\
& \quad j=1,2, \ldots, N_{t}, k=1,2, \ldots, N_{t},
\end{aligned}
$$

where $\mathbf{h}_{j}$ and $\mathbf{h}_{k}$ are two transmit vectors which are the columns of channel matrix $\mathbf{H}(i)$.

Firstly, we consider a set $S=\Phi$ and $L$ transmit vectors with the largest Frobenius norm, as the benchmark

$$
p=\arg \max \left\|\mathbf{h}_{k}\right\|_{F}, \quad k=1,2, \ldots, N_{t}, S=\{p\}
$$

and classify the remaining channel columns into these $L$ selected vectors based on their angles as

$$
\cos \theta_{p, k}=\frac{\left|\mathbf{h}_{p}^{\mathbf{H}} \mathbf{h}_{k}\right|}{\left\|\mathbf{h}_{p}\right\|_{F}\left\|\mathbf{h}_{k}\right\|_{F}}, \begin{aligned}
& \\
& k \in\left\{1,2, \ldots, N_{t}\right\}-S, p \in S .
\end{aligned}
$$

Assume that the first $L$ selected transmit vectors are $\mathbf{h}_{l}, l=$ $1, \ldots, L$. Then the columns of channel matrix can be divided into $L$ subsets as $A_{l}, l=1, \ldots, L$. For each subset $A_{l}$, both norms and the angles between the earlier selected transmit vector $l$ and the rest of $A_{l}$ are taken into consideration.

We define two sets as $S_{A_{l}}$ and $T_{A_{l}}$, which represent the selected and the overall antenna indexes, respectively. Then the numbers of the elements in the two sets are defined as

$$
\begin{aligned}
& \left|S_{A_{l}}\right|=0, \\
& \left|T_{A_{l}}\right|=\left|A_{l}\right|,
\end{aligned}
$$

In the first step, the first selected antenna is the column with the largest Frobenius norm, as $\mathbf{h}_{l}, l=1, \ldots, L$, then it is added into $S_{A_{l}}$ as

$$
\begin{aligned}
& S_{A_{l}}=\left\{\mathbf{h}_{l}\right\}, \\
& T_{A_{l}}=A_{l}-S_{A_{l}}, \\
& \quad l=1, \ldots, L .
\end{aligned}
$$

Therefore, there are still $\left|A_{l}\right|-1$ elements in $T_{A_{l}}$. Furthermore, in the $n$th $(n \geqslant 2)$ step, we calculate the angles between the $(n-1)$ th antennas in $S_{A_{l}}$ and the remaining antennas in $T_{A_{l}}$ and choose the antenna which satisfies

$$
p_{n}=\arg \max _{k \in T_{A_{l}}}\left(\left(\min _{j \in S_{A_{l}}} \theta_{j, k}\right)\left\|\mathbf{h}_{k}\right\|_{F}\right) .
$$

Thus $\mathbf{h}_{p_{n}}$ is added into $S_{A_{l}}$. The above procedures will continue until $n=K_{l}$ for each subset $A_{l}$;

$$
S_{A_{l}}=\left\{\mathbf{h}_{l}, \mathbf{h}_{p_{n}}\right\}, \quad n=2, \ldots, K_{l},\left|S_{A_{l}}\right|=K_{l} .
$$

Finally, the desired selected antennas $N_{s}$ are expressed as

$$
N_{s}=\sum_{l=1}^{L} S_{A_{l}}
$$

In general, the proposed TAS scheme can be summarized in Algorithm 1.

3.2. Threshold-Based NAG-SD Scheme. In this subsection, we show that the complexity of the proposed NAG-SD TAS scheme can be further reduced by introducing a preset threshold. We first analyze the distribution of the Frobenius norms of the transmit vectors for further simplified processing. Since each entry $h_{l j}$ is an i.i.d. random variable with distribution $C N(0,1), \mathbf{h}_{i}$ is an $N_{r}$-dimention transmit vector, $i=1,2, \ldots, N_{t}$; then $\left\|\mathbf{h}_{i}\right\|_{F}^{2}$ follows a chi-square distribution with $2 N_{r}$ degrees of freedom. Thus its probability density function (PDF) is given by

$$
f_{\chi^{2}\left(2 N_{r}\right)}(x)= \begin{cases}\frac{1}{2^{N_{r} \Gamma\left(N_{r}\right)}} e^{-x / 2} x^{N_{r}-1} & x>0, \\ 0 & x \leq 0 .\end{cases}
$$

It is noted that when $\chi^{2}$ is larger than $\chi^{2}\left(2 N_{r}\right)$ and less than $\chi^{2}\left(2 \times\left(2 N_{r}\right)\right)$, the relationship between the PDF and $\chi^{2}$ is almost linear as shown in Figure 1. This observation motivates us to model this relationship as

$$
R(x, P): P=\alpha x+C,
$$

where $x$ is the Frobenius norm of channel columns, $P$ indicates the probability of $x$, and $\alpha$ and $C$ are two constants depending on the number of receive antennas $N_{r}$. For example, for a system with $N_{r} \times N_{t}=2 \times 100$ and $L=64$, according to (15) and (16), we have

$$
\begin{aligned}
& \alpha \approx 0.0786, \\
& C \approx 0.2962 .
\end{aligned}
$$


(1) for $k=1: N_{t}$

$\alpha_{k}=\left\|\mathbf{h}_{k}\right\|_{F}^{2}$

end

$p=\max a_{k}$ until $p=L$

(2) for $i=1: L$

for $j=L+1: N_{t}$

$n(i, j-L)=\frac{\left|\left\langle\mathbf{h}_{i}, \mathbf{h}_{j}\right\rangle\right|}{\alpha_{i} \cdot \alpha_{j}}$

end

end

$m_{l}=$ length $($ find $(\min [n]==l)), l=1, \ldots, L$

$A_{l}=\operatorname{round}\left(m_{l}\left(\frac{N_{s}-L}{N_{t}-L}\right)\right)$

(3) $\sum_{i=1}^{L}\left|A_{l}\right|=N_{t}, S_{A_{l}}=\emptyset, T_{A_{l}}=\left\{1,2, \ldots,\left|A_{l}\right|\right\}$

(4) $p_{l_{1}}=\arg \max _{j \in A_{l}}\left\|h_{j}\right\|_{F}^{2}=\left\|\mathbf{h}_{l}\right\|_{F}^{2}$

(5) for $n=2: K_{l}$

$S_{A_{l}}=S_{A_{l}}+\left\{p_{l_{n-1}}\right\}, T_{A_{l}}=T_{A_{l}}-\left\{p_{l_{n-1}}\right\}$
$\quad$ for all $i \in T_{A_{l}}$

$$
\beta_{p_{l_{n-1}, i}}=\frac{\left|\left\langle\mathbf{h}_{i}, \mathbf{h}_{p_{l_{n-1}, i}}\right\rangle\right|}{\left\|\mathbf{h}_{i}\right\|_{F} \| \mathbf{h}_{p_{l_{n-1}, i} \|_{F}}}
$$

$$
\beta_{i}=\arg \min \left(a \cos \left(\beta_{p_{l_{n-1}, i}}\right)\right)
$$$$
w_{i}=\beta_{i} \cdot\left\|\mathbf{h}_{i}\right\|_{F}
$$

end

$p_{n}=\arg \max _{i \in T_{A_{l}}} w_{i}$

end

(6) $S_{A_{l}}=S_{A_{l}}+\left\{K_{l}\right\}$

(7) Return $S_{A_{l}}$

(8) For each $A_{l}$, preceed the same Steps (4) (7)

Finally, return $N_{s}=\sum_{l=1}^{L} \mathrm{~S}_{A_{l}}$

Algorithm 1: The procedure of NAG-SD TAS scheme.

The probability $P$ can be considered as the ratio of the number of selected antennas to the total number of antennas. For instance, a given

$$
\begin{aligned}
& P=\frac{L}{N_{t}}=0.64, \\
& x=\frac{(P-C)}{\alpha}=2.12
\end{aligned}
$$

is determined as a threshold of $\left\|\mathbf{h}_{i}\right\|_{F}^{2}$ for selecting the large Frobenius norms of the transmit vectors. Then the proposed scheme can be described as the following steps. The first stage invokes almost the same procedure, as described COAS above, that is, calculating the Frobenius norms of the channel columns and comparing them with the threshold $x$. If $\left\|\mathbf{h}_{i}\right\|_{F}^{2} \geqslant$ $x$, the $i$ th antenna is then selected. Assume that $L^{\prime}$ antennas are selected. There may be a discrepancy between $L^{\prime}$ and the desired $L$, written as $\Delta=\left|L-L^{\prime}\right|$, so we randomly increase or decrease $\Delta$ to obtain the required number of antennas $L$.

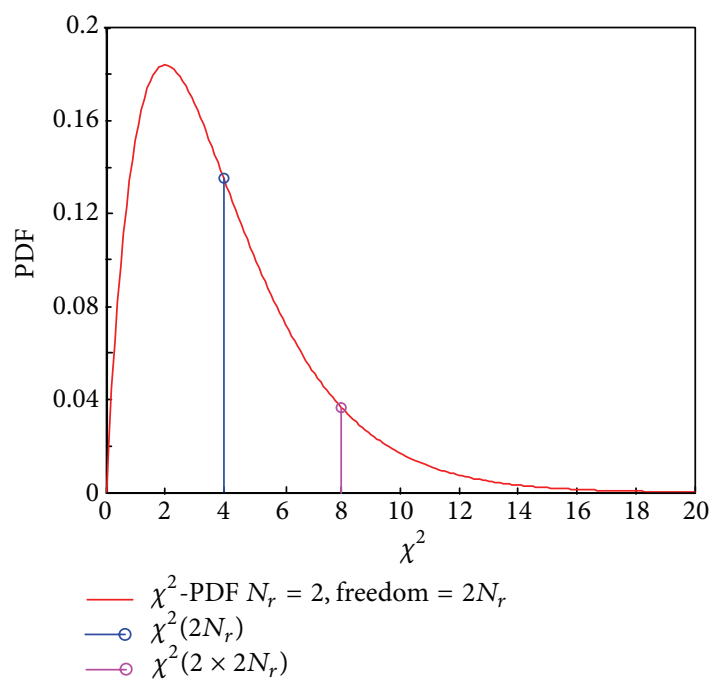

FIGURE 1: PDF of the channel columns with Frobenius norm.

The threshold-based NAC-SD scheme invokes almost the same procedure as described scheme above, except the first step. In the first step, we calculate the Frobenius norm of transmit vectors and judge whether it is higher than the preset threshold. If $\left\|\mathbf{h}_{l}(i)\right\|_{F}^{2} \geq x$, the $l$ th antenna will be selected. Assuming $K$ antennas are selected in the first step, the remaining $\left(N_{t}-K\right)$ antennas will be selected and processed as the processing of NAC-SD TAS presented in Algorithm 1.

3.3. Complexity Analysis. In this section, the computational complexity is analyzed and counted as the number of real floating point operations (flops). For the proposed NAGSD scheme, computing the Frobenius norm for the column vectors $\left\|\mathbf{h}_{i}(i)\right\|_{F}^{2}, i=1,2, \ldots, N_{t}$, will take $4 N_{t} N_{r}$ flops. And calculating the angle between two columns $\mathbf{h}_{i}(i)$ and $\mathbf{h}_{j}(i)$ needs $10 N_{r}+3$ flops, so the number of the angle calculations is given as

$$
\left(10 N_{r}+3\right)\left(N_{s}-i\right)+\left(N_{s}-i\right), \quad i=1,2, \ldots, L-1 .
$$

Therefore, the total number of flops for NAG-SD is computed as

$$
\begin{aligned}
\varphi_{1} & =\sum_{l=1}^{L}\left\{4 N_{t_{l}} N_{r_{l}}\right. \\
& \left.+\sum_{i=1}^{K_{l}}\left[\left(10 N_{r_{l}}+3\right)\left(K_{l}-i\right)+\left(K_{l}-i\right)\right]\right\} \\
& =\sum_{l=1}^{L}\left\{4 N_{t_{l}} N_{r_{l}}\right. \\
& \left.+\sum_{i=1}^{K_{l}}\left[\left(10 N_{r_{l}}+3\right)\left(N_{t_{l}}-\frac{K_{l}}{2}\right)+\left(K_{l}-1\right)\right]\right\},
\end{aligned}
$$

where $N_{t_{l}}, N_{r_{l}}$, and $K_{l}$ represent the number of transmit antennas, receive antennas, and the desired antennas for each subset $A_{l}$, respectively. 
TABLE 1: Computational complexity comparisons.

\begin{tabular}{lccccr}
\hline TAS method & EDAS & EDAS-RLC & COAS & NAG-SD & Threshold-based NAG-SD \\
\hline Computational complexity (flops) & $7.6304 \times 10^{19}$ & 209217 & 682 & 7900 & 2778 \\
\hline
\end{tabular}

Similarly, the computational complexity for thresholdbased NAC-SD can also be computed. Firstly, consider that the flops of threshold-based NAC-SD are statistics. If $\left\|\mathbf{h}_{l}(i)\right\|_{F}^{2} \geq x$, the $l$ th antenna will be selected; then the number of flops is written as

$$
\varphi_{2,1}=4 N_{t_{l}} N_{r_{l}}, \quad l=1,2, \ldots, L .
$$

In case of $\left\|\mathbf{h}_{l}(i)\right\|_{F}^{2}<x$, the selection processing is the same as NAG-SD. In general, the total number of flops for threshold-based NAG-SD is given as

$$
\begin{aligned}
\varphi_{2}= & \varphi_{2,1}+\sum_{i=1}^{K_{l}}\left[\left(10 N_{r_{l}}+3\right)\left(K_{l}-i\right)+\left(K_{l}-i\right)\right] \\
= & 4 N_{t_{l}} N_{r_{l}} \\
& +\sum_{i=1}^{K_{l}}\left[\left(10 N_{r_{l}}+3\right)\left(N_{t_{l}}-\frac{K_{l}}{2}\right)+\left(K_{l}-1\right)\right], \\
& l=1,2, \ldots, L .
\end{aligned}
$$

According to the above analysis, we give a further example to compare the complexity of current TAS schemes. Assuming a SM-MIMO system with $N_{t} \times N_{r}=50 \times 2, N_{s}=$ 32 , and $L=4$, the comparisons among current schemes in computational complexity are presented in Table 1. In general, we show that the proposed schemes can considerably reduce the computational complexity compared to the original methods.

\section{Simulation Results}

In this section, we validate the proposed TAS schemes for SM systems in large-scale transmit antenna configuration and compare them with conventional ED-based and COAS TAS methods. We assume that an equal power per active transmit antenna is allocated, and the channel state information of Rayleigh flat fading channel is perfectly estimated at the receiver. An optimal ML detector is deployed at the receiver.

Figures 2 and 3 show the BER performance of the proposed NAG-SD, threshold-based NAC-SD, COAS, and that of the conventional SM under different conditions. We consider a large-scale SM-MIMO system employing $N_{t}=$ $50,100, N_{r}=2$, and $N_{s}=32,64$ with QPSK modulation. For reference, the performance of conventional SM is also included. It can be observed from Figure 2 that the proposed two TAS schemes outperform the conventional SM scheme and COAS by $2.5 \mathrm{~dB}$ at a BER of $10^{-3}$, respectively. The computational complexity is also studied and counted as shown in Figure 4. In general, simulation results show that the proposed schemes can make a balanced tradeoff between transmission performance and computational complexity, compared to traditional TAS schemes.

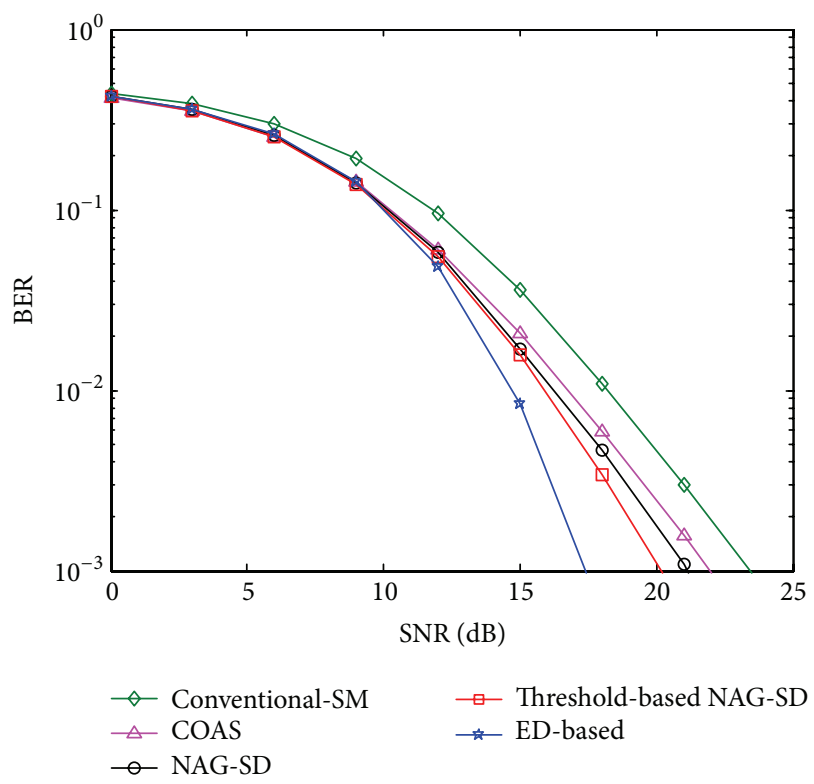

FIGURE 2: BER performance of different TAS schemes in SM-MIMO with $N_{t}=50, N_{r}=2$, and $N_{s}=32$.

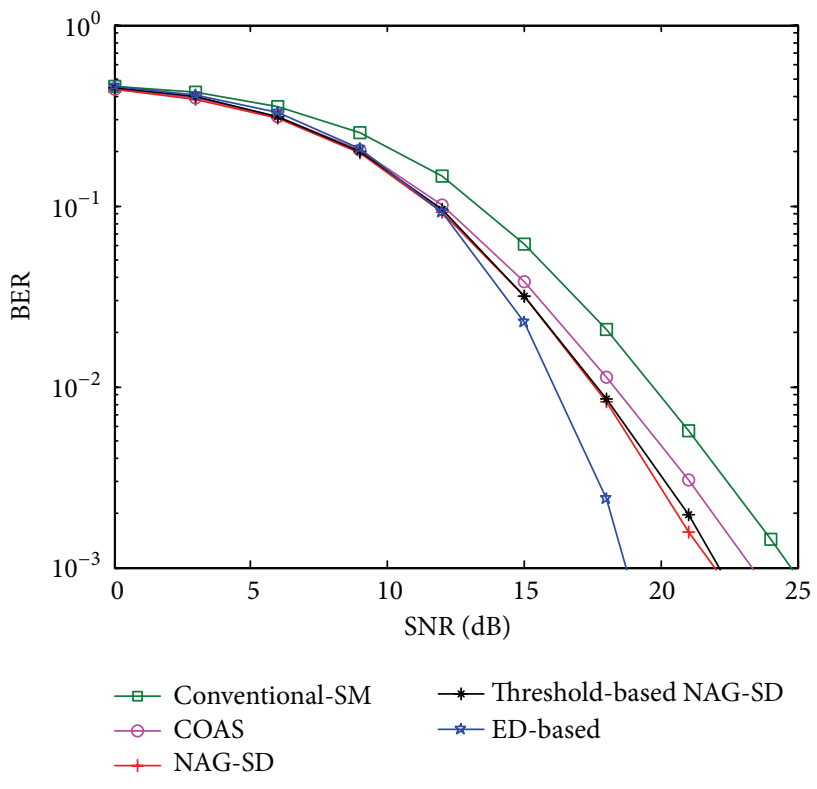

FIGURE 3: BER performance of different TAS schemes in SM-MIMO with $N_{t}=100, N_{r}=4$, and $N_{s}=64$.

\section{Conclusion}

In this paper, we proposed a class of novel TAS schemes, named as norm-angle guided subset division and thresholdbased NAG-SD ones, for spatial modulation combined largesale MIMO environments. We showed that the proposed 


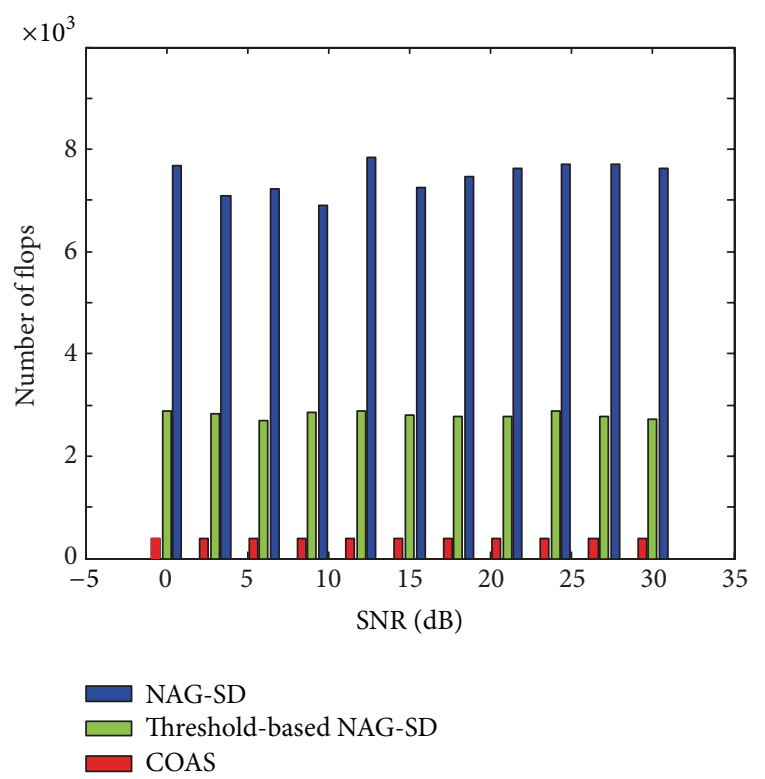

FIGURE 4: Computational complexity comparison of different TAS schemes in SM-MIMO.

schemes achieve a considerable reduction in implementation complexity than conventional suboptimal ones in the TAS for large-scale SM. Meanwhile, the proposed schemes were shown to be capable of achieving about 2-3 dB at BER of $10^{-3}$ compared to conventional schemes. In general, the proposed schemes can be used in SM combined large-scale MIMO for future $5 \mathrm{G}$ wireless communications, by making a balanced tradeoff between transmission performance and computational complexity.

\section{Conflict of Interests}

The authors declare that there is no conflict of interests regarding the publication of this paper.

\section{Acknowledgments}

This work was supported in part by the National Science Foundation of China under Grant no. 61471090, the open research fund of National Mobile Communications Research Laboratory, Southeast University (no. 2013D05), the Foundation Project of National Key Laboratory of Science and Technology on Communications under Grant 9140C020108140C02005, and the National High-Tech R\&D Program of China ("863" Project under Grant no. 2015AA01A709).

\section{References}

[1] M. D. Renzo, H. Haas, A. Ghrayeb, S. Sugiura, and L. Hanzo, "Spatial modulation for generalized MIMO: challenges, opportunities, and implementation," Proceedings of the IEEE, vol. 102, no. 1, pp. 56-103, 2014.
[2] M. D. Renzo, H. Haas, and P. M. Grant, "Spatial modulation for multiple-antenna wireless systems: a survey," IEEE Communications Magazine, vol. 49, no. 12, pp. 182-191, 2011.

[3] P. Yang, M. D. Renzo, Y. Xiao, S. Li, and L. Hanzo, "Design guidelines for spatial modulation," IEEE Communications Surveys and Tutorials, vol. 17, no. 1, pp. 6-26, 2015.

[4] J. Hoydis, S. Ten Brink, and M. Debbah, "Massive MIMO in the UL/DL of cellular networks: how many antennas do we need?" IEEE Journal on Selected Areas in Communications, vol. 31, no. 2, pp. 160-171, 2013.

[5] F. Rusek, D. Persson, B. K. Lau et al., "Scaling up MIMO: opportunities and challenges with very large arrays," IEEE Signal Processing Magazine, vol. 30, no. 1, pp. 40-60, 2013.

[6] K. Zheng, L. Zhao, J. Mei, B. Shao, W. Xiang, and L. Hanzo, "Survey of large-scale MIMO systems," IEEE Communications Surveys \& Tutorials, vol. 17, no. 3, pp. 1738-1760, 2015.

[7] T. Luan, F. Gao, and X.-D. Zhang, "Joint resource scheduling for relay-assisted broadband cognitive radio networks," IEEE Transactions on Wireless Communications, vol. 11, no. 9, pp. 3090-3100, 2012.

[8] F. Zhu, F. Gao, M. Yao, and H. Zou, "Joint information- and jamming- beamforming for physical layer security with full duplex base station," IEEE Transactions on Signal Processing, vol. 62, no. 24, pp. 6391-6401, 2014.

[9] K. Zheng, L. Zhao, J. Mei, M. Dohler, W. Xiang, and Y. Peng, "10 $\mathrm{Gb} / \mathrm{s}$ hetsnets with millimeter-wave communications: access and networking-challenges and protocols," IEEE Communications Magazine, vol. 53, no. 1, pp. 222-231, 2015.

[10] K. Zheng, X. Zhang, Q. Zheng, W. Xiang, and L. Hanzo, "Quality-of-experience assessment and its application to video services in LTE networks," IEEE Wireless Communications, vol. 22, no. 1, pp. 70-78, 2015.

[11] P. Yang, Y. Xiao, L. Li, Q. Tang, Y. Yu, and S. Li, "Link adaptation for spatial modulation with limited feedback," IEEE Transactions on Vehicular Technology, vol. 61, no. 8, pp. 38083813, 2012.

[12] R. Rajashekar, K. V. S. Hari, and L. Hanzo, "Antenna selection in spatial modulation systems," IEEE Communications Letters, vol. 17, no. 3, pp. 521-524, 2013.

[13] N. Pillay and X. U. Hongjun, "Comments on antenna selection in spatial modulation systems," IEEE Communications Letters, vol. 17, no. 9, pp. 1681-1683, 2013.

[14] X. Wu, M. Di Renzo, and H. Haas, "Direct transmit antenna selection for transmit optimized spatial modulation," in Proceedings of the 78th IEEE Vehicular Technology Conference (VTC Fall '13), pp. 1-5, IEEE, Las Vegas, Nev, USA, September 2013.

[15] K. Ntontin, M. D. Renzo, A. I. Perez-Neira, and C. Verikoukis, "A low-complexity method for antenna selection in spatial modulation systems," IEEE Communications Letters, vol. 17, no. 12, pp. 2312-2315, 2013.

[16] L. Li, Y. Xiao, W. Li, and S. Li, "Spatial modulation method based on transmitting antenna selection for MIMO (MultiInput Multi-Output) system," CN 201210179492, 2012.

[17] Z.Zhou, N. Ge, and X. Lin, "Reduced-complexity antenna selection schemes in spatial modulation," IEEE Communications Letters, vol. 18, no. 1, pp. 14-17, 2014.

[18] J. Wang, S. Jia, and J. Song, "Signal vector based detection scheme for spatial modulation," IEEE Communications Letters, vol. 16, no. 1, pp. 19-21, 2012. 

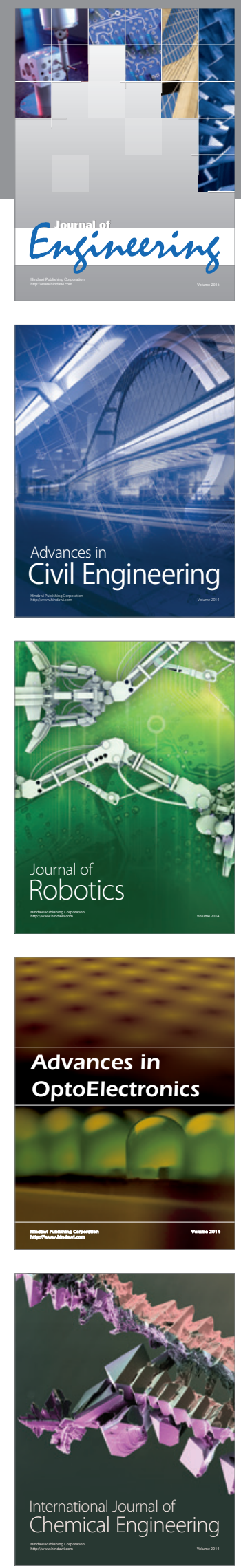

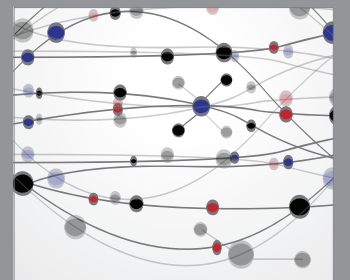

The Scientific World Journal
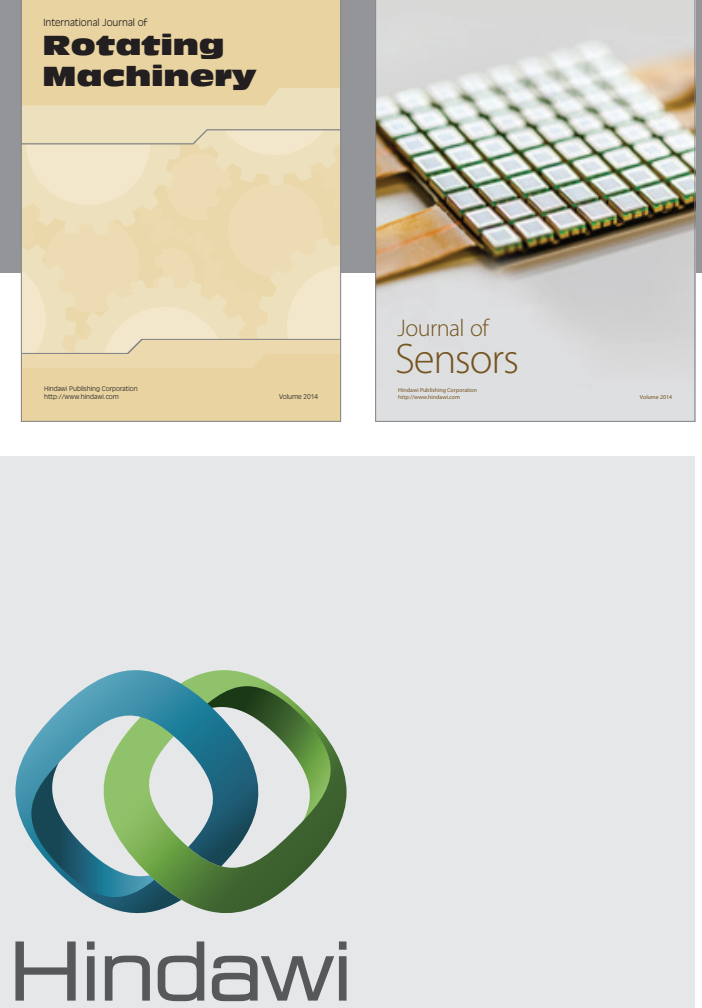

Submit your manuscripts at http://www.hindawi.com
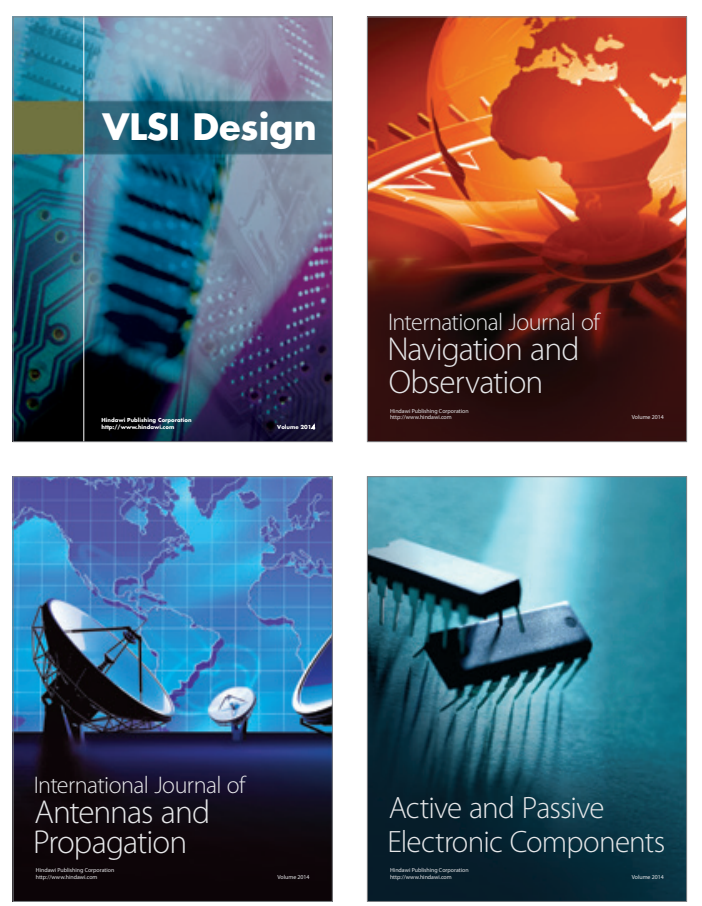
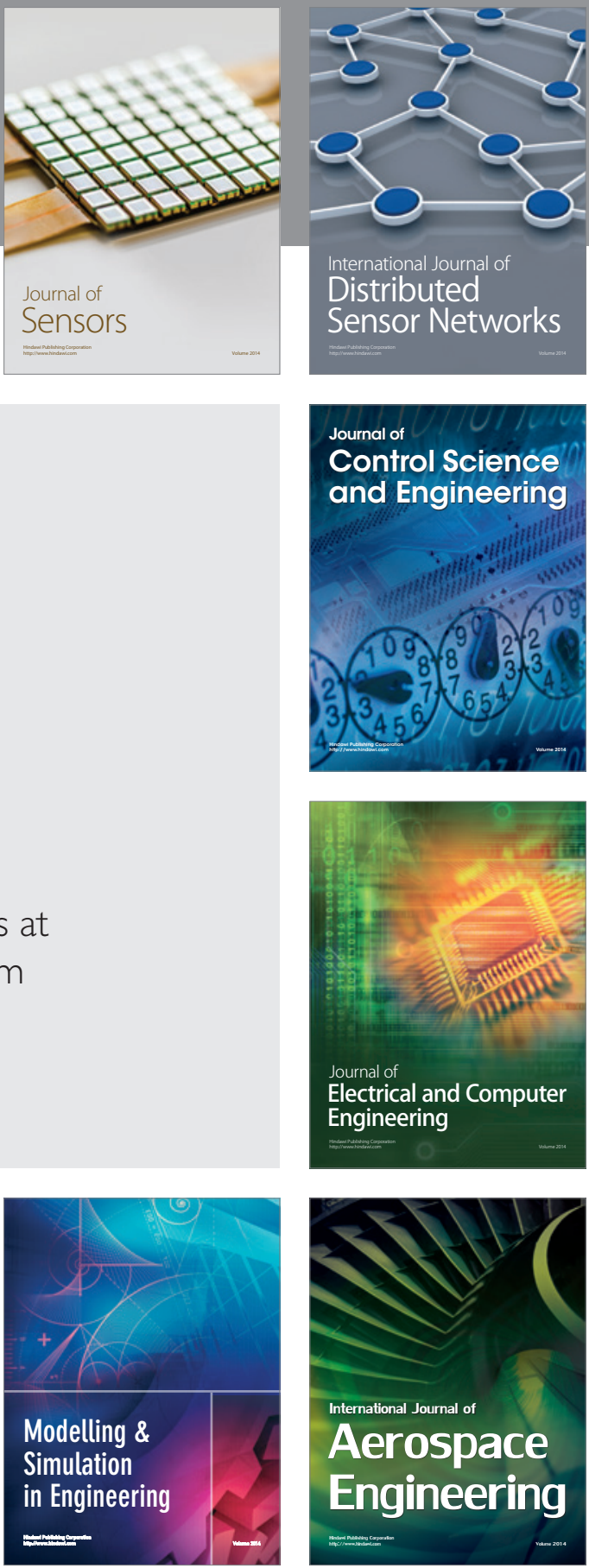

Journal of

Control Science

and Engineering
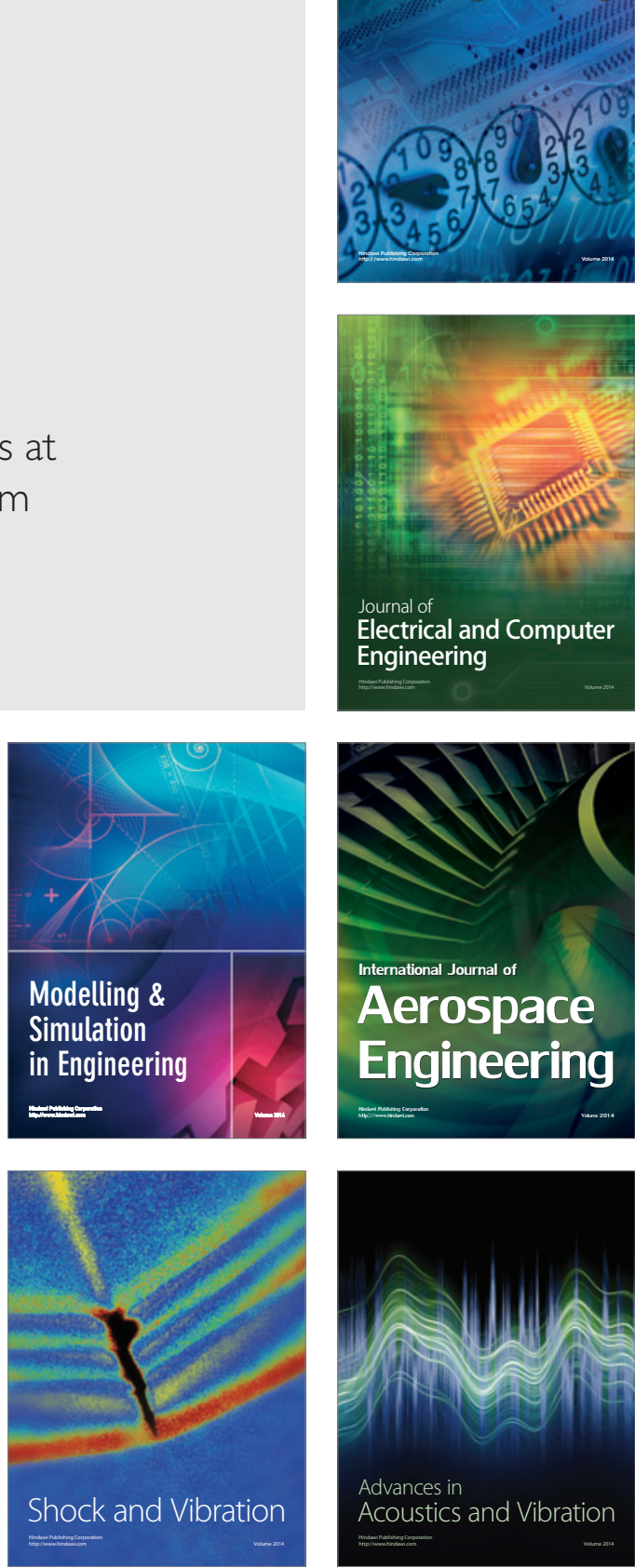